\title{
Íleo biliar do cólon sigmóide: uma causa rara de obstrução intestinal
}

\author{
Gallstone ileus of the sigmoid colon: a rare cause of intestinal obstruction \\ Ileo biliar de colon sigmoideo: una rara causa de obstrucción intestinal
}

Aurélio Rocha Batista de Oliveira ${ }^{1 *}$, Amanda Lage Araújo Alves², Amanda Machado Ferreira², Nájila Aélida Oliveira Viana², Raissa Dalat Coelho Furtado², Rafael Bruno da Silveira Alves², Tatiana Dalat Coelho Furtado³.

\section{RESUMO}

Objetivo: Mostrar a importância de se considerar o íleo biliar como uma causa de abdome agudo obstrutivo. Detalhamento do caso: Paciente sexo masculino, 77 anos, admitido em unidade hospitalar com queixa de dor e distensão abdominal, vômitos e constipação. Colelitíase diagnosticada há 2 anos, sem tratamento. Realizou tomografia computadorizada de abdome e pelve que identificou distensão hidroaérea de intestino delgado e dilatação com retenção de fezes e líquidos dos cólons à montante de uma formação com configuração arredondada, densidade heterogênea, situada na porção proximal do sigmoide, medindo $3,3 \mathrm{~cm}$ no maior diâmetro. Além disso, foi identificado ar na região da vesícula biliar, vias biliares e colédoco, com trajeto fistuloso bem definido entre o ângulo hepático do cólon e o leito da vesícula biliar. Fechado diagnóstico de abdome agudo obstrutivo devido a íleo biliar de cólon sigmoide. Realizada laparotomia com colecistectomia, colectomia direita e remoção do cálculo impactado. Considerações finais: $O$ íleo biliar do cólon é uma causa rara de obstrução mecânica em intestino grosso, causada pela impactação de cálculo biliar em ponto de estreitamento colônico e deve ser considerado como causa de abdome agudo principalmente em idosos. A cirurgia é a base do seu tratamento.

Palavras-chaves: Íleo biliar, Obstrução intestinal, Abdome agudo.

\begin{abstract}
Objective: To show the importance of considering the gallstone ileus as a cause of acute obstructive abdomen. Case details: Male patient, 77 years old, admitted in hospital unit with complaints of abdominal pain and distension, vomiting and constipation. Cholelithiasis diagnosed 2 years ago, without treatment. CT scan of the abdomen and pelvis identified hydroaereal distension of small intestine loops and dilation with retention of feces and colon fluids upstream of a rounded configuration, with heterogeneous density, located in the proximal portion of the sigmoid, with $3.3 \mathrm{~cm}$ in the largest diameter. Presence of diverticula along the colon, giving a spiky aspect to the intestinal walls, parietal hypertrophy and luminal reduction. Air in the region of the gallbladder as well as in the bile ducts and choledochus. Well-defined fistulous tract between the hepatic angle of the colon and the bed of the gallbladder. Closed diagnosis of acute obstructive abdomen due to gallstone ileus of the sigmoid colon. A laparotomy was performed with cholecystectomy and colectomy in the middle third of the transverse colon. Final considerations: The colonic gallstone ileus is a rare cause of mechanical obstruction of the large intestine, usually caused by impaction of gallstones at the point of colonic narrowing. Surgery is the basis of treatment.
\end{abstract}

Keywords: Gallstone ileus, Bowel obstruction, Acute abdomen.

\section{RESUMEN}

Objetivo: Mostrar la importancia de considerar el íleon biliar como causa de abdomen obstructivo agudo. Detalles del caso: Paciente sexo masculino, 77 años, ingressa en el hospital con quejas de dolor y distensión abdominal, vómitos y estreñimiento. Colelitiasis diagnosticada hace 2 años, sin tratamiento. La tomografía computarizada de abdomen y pelvis identificó hidro distensión de intestino delgado y dilatación con retención de heces y líquidos del colon aguas arriba de una formación con configuración redondeada,

\footnotetext{
${ }^{1}$ Hospital Nossa Senhora da Conceição, Pará de Minas - MG. *E-mail: aureliorocha13@hotmail.com

2 Hospital Alberto Cavalcanti, Belo Horizonte - MG.

${ }^{3}$ Universidade Federal de Ouro Preto (UFOP), Ouro Preto - MG.
}

SUBMETIDO EM: 5/2021

ACEITO EM: 5/2021

PUBLICADO EM: 6/2021 
densidad heterogénea, ubicada en la porción proximal del sigmoideo, midiendo $3.3 \mathrm{~cm}$ en el diámetro mayor. Además, se identificó aire en la región de la vesícula biliar, en los conductos biliares y colédoco con trayectoria fistulosa bien definida entre el ángulo hepático del colon y el lecho de la vesícula biliar. Diagnóstico de cierre es de abdomen obstructivo agudo por íleon biliar del colon sigmoide. Se realizó una laparotomía con colecistectomía, colectomía derecha y eliminación del cálculo impactado. Consideraciones finales: El íleon biliar colónico es una causa poco común de obstrucción mecánica del intestino grueso, causada por la impactación de cálculos biliares en el punto de estrechamiento del colon y debe considerarse como una causa de abdomen agudo principalmente en los ancianos. La cirugía es la base del tratamiento.

Palabras clave: Íleo biliar, Obstrucción intestinal, Abdomen agudo.

\section{INTRODUÇÃO}

O íleo biliar (IB) é uma causa extremamente rara de obstrução mecânica do intestino grosso, responsável por 2 a $8 \%$ dos casos. Ocorre devido à impactação de grande cálculo biliar em um ponto de estreitamento, devido a uma fístula colecistocolônica. Um fator predisponente para esta patologia é a ocorrência de episódios recorrentes de inflamação da vesícula biliar. A conexão fistulosa se forma após um episódio de colecistite aguda que leva à formação de aderências entre a vesícula biliar e o cólon. Esse tipo de obstrução tende a ocorrer onde há patologia preexistente, em local de subestenose secundária a doença diverticular ou a irradiação pélvica anterior, uma vez que o diâmetro normal do cólon permite a passagem livre desses cálculos (ALENCASTRO MC, et al., 2013; OSMAN N, et al., 2010).

É uma doença de alta morbimortalidade devido ao desafio diagnóstico, à sua apresentação tardia e às comorbidade geralmente associadas, que afetam principalmente pacientes idosos e mulheres (ANSELINE P, 1981; CARLSSON T e GANDHI S, 2015). Os sinais e sintomas normalmente são inespecíficos e podem levar a atrasos diagnósticos. Mais de $50 \%$ dos casos são descobertos apenas durante a abordagem cirúrgica devida ao quadro de abdome agudo (CARLSSON T e GANDHI S, 2015). A maioria dos pacientes com IB colônico apresenta sintomas de obstrução do intestino grosso, no entanto apenas $25 \%$ têm história de cólica biliar no ano anterior (RANGA N, 2011).

O uso de exame de imagem exploratório do abdome melhorou a precisão diagnóstica e a velocidade das decisões cirúrgicas para maximizar um bom prognóstico para o paciente. Menos de $20 \%$ dos cálculos biliares são visíveis nas radiografias abdominais. Com isso, a tomografia computadorizada abdominal com contraste mostra-se uma importante ajuda já que permite melhor visualização do ponto de obstrução e o local da fístula (ALENCASTRO MC, et al., 2013; CHANG L, et al.,2018; OSMAN N, et al., 2010).

O manejo cirúrgico é o tratamento de escolha na maioria dos casos para prevenir as complicações, porém a abordagem cirúrgica ideal não está estabelecida pela literatura científica. A resolução da obstrução intestinal é a base do tratamento, enquanto a necessidade de colecistectomia e de correção da fístula biliar precisa ser analisada com base nas características do paciente (CHANG L, et al., 2018; CLARO M, et al., 2020; SCHOOFS C, et al., 2010).

Neste estudo, objetiva-se relatar um caso incomum de obstrução do intestino grosso como complicação de litíase da vesícula biliar, discutindo as modalidades diagnósticas e terapêuticas dessa rara entidade.

\section{DETALHAMENTO DO CASO}

Trata-se de paciente do sexo masculino, 77 anos, admitido em unidade hospitalar com queixa de dor e distensão abdominal há 20 dias. Inicialmente foi realizado o preenchimento completo da anamnese e do termo de consentimento livre e esclarecido (TCLE), com acompanhamento do familiar responsável, que relatou a nível de complemento dos dados clínicos que o paciente apresentava vômitos esporádicos, constipação intestinal e piora da dor nos últimos dias, se localizando principalmente em abdome inferior. Portador de Diabetes Mellitus tipo 2, hipertensão arterial sistêmica e previamente submetido à prostatectomia devido a hiperplasia prostática benigna. Colelitíase diagnosticada há 2 anos, sem tratamento cirúrgico realizado. Ainda, foi esclarecido que os dados clínicos, laboratoriais e documentação radiológica do caso clínico/cirúrgico do paciente seria utilizados para fins de publicação em revista científica. 
Ao exame físico de admissão encontrava-se corado, desidratado, eucárdico, eupneico em ar ambiente, afebril, com abdome distendido, hipertimpânico e doloroso à palpação difusamente, especialmente em abdome inferior. Toque retal com ampola retal ampla, dedo de luva com fezes, sem outros achados. Exames laboratoriais apontaram hipocalemia, sem demais alterações.

A tomografia computadorizada de abdome e pelve (Figuras 1 e 2) identificou importante distensão hidroaérea de alças de intestino delgado e dilatação com retenção de fezes e líquidos dos cólons à montante de formação de configuração arredondada, com densidade heterogênea, situada na porção proximal do sigmoide, medindo $3,3 \mathrm{~cm}$ no maior diâmetro. Presença de divertículos ao longo do cólon, conferindo um aspecto espiculado das paredes, hipertrofia parietal e redução luminal. Ar na região da vesícula biliar bem como em vias biliares e colédoco. Trajeto fistuloso bem definido entre o ângulo hepático do cólon e o leito da vesícula biliar.

Figura 1- Tomografia de abdome e pelve: cálculo biliar impactado em topografia de colón sigmoide.

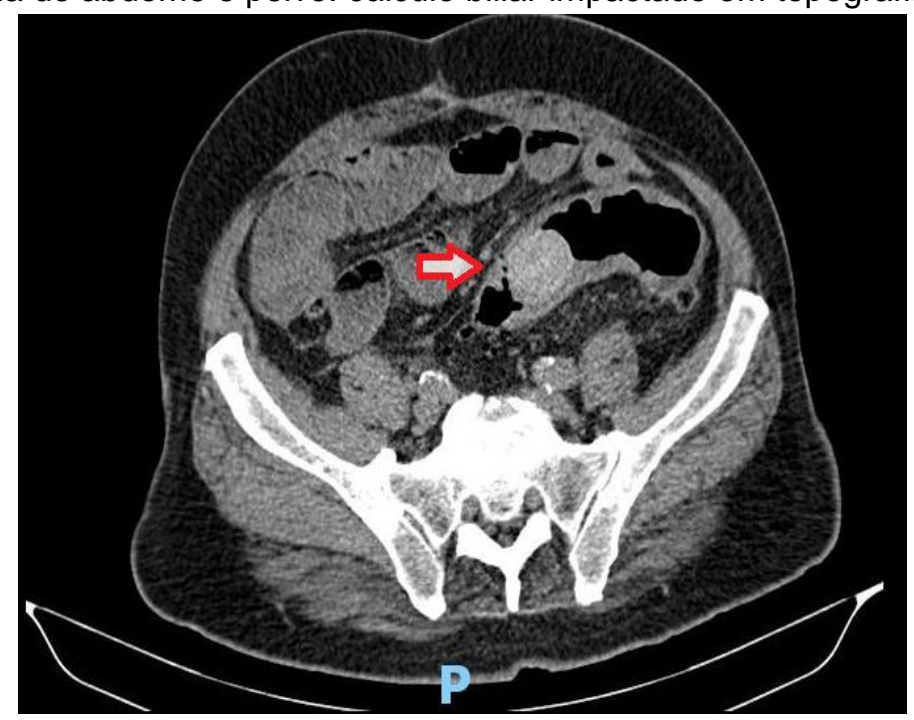

Legenda: P: posterior; Seta: cálculo biliar impactado em cólon sigmoide.

Fonte: Oliveira ARB, et al., 2021.

Figura 2- Tomografia de abdome e pelve: cálculo biliar impactado em topografia de colón sigmoide.

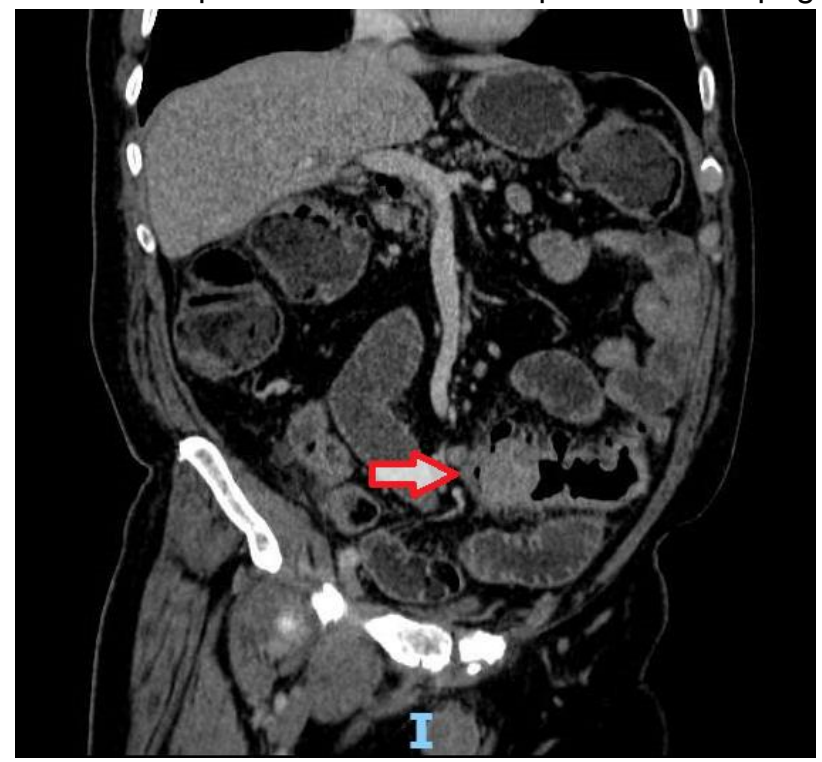

Legenda: I: inferior; Seta: cálculo biliar impactado em cólon sigmoide.

Fonte: Oliveira ARB, et al., 2021. 
Nesse contexto, o paciente foi submetido à laparotomia exploradora. Foi detectado pneumoperitôneo com grande quantidade de líquido livre purulento em cavidade abdominal, perfuração de cólon ascendente, intenso processo inflamatório com múltiplas aderências em topografia da vesícula biliar e cálculo impactado em cólon sigmoide. Optado por realização de colecistectomia e colectomia direita até terço médio do cólon transverso com ordenha e remoção do cálculo que se encontrava no sigmoide (Figura 3). Confeccionada ileostomia com fístula mucosa de transverso, mantido dreno tubular para vigilância em topografia biliar. A recuperação pós-operatória foi conduzida em unidade de terapia intensiva, no entanto o paciente evoluiu a óbito dias após o procedimento devido à intensa resposta inflamatória relacionada ao trauma cirúrgico.

Figura 3- Cálculo biliar removido

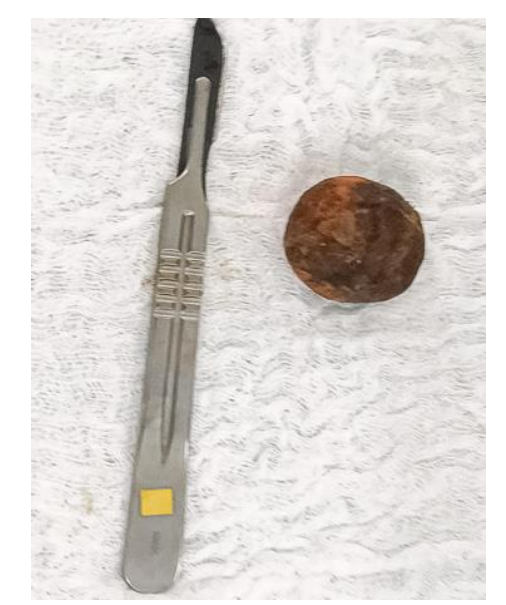

Fonte: Oliveira ARB, et al., 2021.

\section{DISCUSSÃO}

O IB é uma complicação rara da colelitíase causado por comunicação anômala entre a via biliar e o trato gastrointestinal (TGI). Essa fistulização se dá geralmente a partir de quadros inflamatórios recorrentes da vesícula biliar, que adere a estruturas abdominais adjacentes permitindo a mobilização e impactação de cálculos no TGI (ALENCASTRO MC, et al., 2013; HALABI WJ, et al., 2014; LAXAGUE F, et al., 2020; OSMAN N, et al., 2010; SCHOOFS C, et al., 2010). Ainda mais rara é a ocorrência da fístula acometendo 0 cólon (fístula colecistocolônica) como a encontrada no caso descrito, correspondente a 2 a $8 \%$ dos casos. De acordo com dados científicos, na maior parte das vezes (65-75\%) as fístulas colecistoentéricas acometem o duodeno e o cálculo se impacta preferencialmente no íleo (70\%) (CUADRA MB, et al., 2017; FARKAS N, et al., 2018; RIGLER LG, et al., 1941).

Epidemiologicamente, o caso relatado apresenta conformidade com a literatura científica devido a tratarse de indivíduo com mais de 60 anos de idade, portador de comorbidades crônicas inclusive intestinal (diverticulose), porém diverge em relação ao sexo pois o IB é mais frequente entre o sexo feminino $(80 \%$ das vezes) (ANSELINE P, 1981; CLAVIEN P, et al., 1990; INUKAI K, 2019; LAXAGUE F, et al., 2020; SCHOOFS C, et al., 2010).

O diagnóstico de IB deve sempre ser suspeitado frente a um quadro obstrutivo em pacientes idosos, havendo ou não história prévia de cólicas biliares recorrentes. Pode apresentar-se com sintomas de obstrução do TGI alto (vômitos biliosos precoces e distensão gástrica) ou baixo (dor e distensão abdominal, náuseas, parada de eliminação de flatos e fezes, vômito fecalóide) a depender do sítio de impactação do cálculo (CLAVIEN P, et al., 1990; INUKAI K, et al., 2018; INUKAI K, 2019). No caso de fístulas colecistocolônicas os pacientes podem ainda manifestar sintomas não específicos de diarreia (devido ao efeito laxativo dos ácidos biliares) e colangite (devido a refluxo de conteúdo fecal para o trato biliar) (ANSELINE P, 1981; CLARO M, et al., 2020; HALABI WJ, et al., 2014; RIGLER LG, et al., 1941; TAN YM, et al., 2004). 
O raio-X de abdome pode mostrar ar na topografia da vesícula e via biliar (aerobilia), sinais de obstrução intestinal parcial ou completa e a localização de um cálculo radio-opaco em local aberrante. A tomografia abdominal pode orientar o diagnóstico se caracterizar aerobilia e cálculo impactado, e o estudo contrastado do tubo digestivo pode mostrar fístula bilio-digestiva e localizar o cálculo no segmento do TGI. (CLAVIEN P, et al., 1990; INUKAI K, 2019).

Rigler et al. descreveram em 1941 uma tríade que compreende sinais radiográficos de distensão do TGl, pneumobilia e identificação de cálculo em topografia distinta da via biliar (RIGLER LG, et al., 1941). Tais sinais são encontrados também à tomografia contrastada de abdomen, hoje em dia considerada o padrão ouro para diagnóstico de imagem do IB, com sensibilidade de 93\% e especificidade de $100 \%$ (CLAVIEN P, et al., 1990; HALABI WJ, et al., 2014; INUKAI K, 2019; LAXAGUE F, et al., 2020; LEE CH, et al., 2015). Dessa forma, a Tríade de Rigler colaborou para o correto diagnóstico clínico pré-operatório do caso relatado. Apesar de ser um achado patognomônico do IB, estima-se que a Tríade seja encontrada somente em menos de 50\% dos casos (CLAVIEN P, et al., 1990; INUKAI K, et al., 2018; INUKAI K, 2019; LAXAGUE F, et al., 2020; RIGLER LG, et al., 1941).

O tratamento de tal enfermidade é essencialmente cirúrgico. Há relatos de tentativa de manejo não operatório em casos de IB com obstrução colônica, resultando em alto índice de falhas (74\%) (CLAVIEN P, et al., 1990; FARKAS N, et al., 2018). São possíveis as abordagens cirúrgicas por vias laparotômica, laparoscópica, colonoscópica, endoscópica e por litotripsia. Os três últimos, por serem menos invasivos, podem ser preferíveis frente a pacientes criticamente debilitados e em idade avançada, porém associam-se a insucesso com maior frequência. (CLAVIEN P, et al., 1990; LAXAGUE F, et al., 2020; INUKAI K, 2019; LEE CH, et al., 2015).

A abordagem cirúrgica deve basear-se no objetivo primário de resolução do quadro obstrutivo. Assim como relatado no caso deste estudo, preconiza-se que nas modalidades de cirurgia laparotômica ou laparoscópica seja realizada a enterolitotomia seguida por enterorrafia e, caso haja isquemia intestinal ou perfuração, pode ser necessária realização de enterectomia (ANSELINE P, 1981; CARLSSON T e GANDHI S, 2018; CLAVIEN P, et al., 1990; INUKAI K, et al., 2018; LEE CH, et al., 2015; REISNER RM e COHEN JR, 1994).

Assim, a abordagem em uma etapa envolve a enterolitotomia, a colecistectomia e a exploração da fístula com ou sem a exploração do colédoco. Tem como principais vantagens a prevenção de colecistite, de colangite e de carcinoma de vesícula biliar, uma vez que a síndrome de Mirizzi comumente associada é fator de risco para neoplasia da vesícula. Entretanto, independente da escolha de abordagem, o tratamento cirúrgico do é normalmente realizado em caráter de urgência (LEE CH, et al., 2015; INUKAI K, 2019).

Permanece ainda controversa e carece de discussões científicas a melhor forma de manejo da fístula colecistoentérica nos casos de IB. Devido à raridade da patologia em questão, não há consenso descrito na literatura científica quanto a relação entre riscos e benefícios da enterolitotomia com colecistectomia e correção da fístula concomitantemente (cirurgia em um único tempo), assim como realizado na ocasião descrita, ou enterolitotomia isolada reservando o manejo da fístula para um segundo momento (CLARO M, et al., 2020; CUADRA MB, et al., 2017; HALABI WJ, et al., 2014; INUKAI K, et al., 2018; REISNER RM e COHEN JR, 1994; PÉREZ JM, et al., 2012).

Estudos sugerem não haver diferença significativa de mortalidade entre as duas modalidades cirúrgicas (respectivamente $16,9 \%$ e $11,7 \%$ ), mas aproximadamente $5 \%$ a $10 \%$ dos paciente recorrem com o quadro caso seja optado por não abordagem imediata da fístula (CLAVIEN P, et al., 1990; HALABI WJ, et al., 2014; TAN YM, et al., 2001). Há ainda estudos que concluíram em recomendação da cirurgia em um único tempo como preferível para pacientes que apresentem fístula do tipo colecistocolônica, assim como neste relato, devido a uma menor taxa de fechamento espontâneo em relação às demais fístulas colecistoentéricas (CLAVIEN P, et al., 1990; INUKAI K, et al., 2018; INUKAI K, 2019; RANGA N, 2011; TAN YM, et al., 2001).

Ao descrever uma condição patológica rara cujo manejo cirúrgico ainda não foi padronizado cientificamente, objetiva-se contribuir com a atual literatura médica. Levando-se em consideração que está 
relacionado a uma condição clínica muito frequente na população mundial (a colelitíase), é importante destacar que deve-se suspeitar do IB diante de pacientes idosos que apresentem sintomas de obstrução intestinal aguda e que os achados em exames de imagem podem desempenhar importante papel para um correto diagnóstico pré-operatório.

Também confere relevância a este relato o fato de na ocasião ter sido optado pelo tratamento cirúrgico com remoção do cálculo impactado e colectomia devido a perfuração intestinal, além de concomitante colecistectomia com correção da fístula colecistoentérica. Apesar de não haver padronização quanto ao tratamento do IB, esta abordagem encontra respaldo científico e neste caso resultou em um desfecho insatisfatório provavelmente devido às condições clínicas prévias desfavoráveis que o paciente apresentava.

O presente relato, portanto, traz à tona a necessidade da inclusão do íleo biliar colônico dentre os possíveis diagnósticos diferencias em um quadro de obstrução intestinal aguda, reforçando o que há de atual e preconizado em relação à propedêutica e terapêutica de uma doença de alta morbimortalidade $e$ desafio diagnóstico. Apresenta-se em conformidade com a literatura e fomenta a necessidade de individualização de cada caso, levando-se em consideração as condições clinicas do paciente, as características do serviço e a experiência do cirurgião assistente. Dessa forma, contribui cientificamente ao demonstrar as incertezas ainda existentes no manejo da doença, impulsionando o aprimoramento clinicocirúrgico através de estudos multicêntricos.

\section{REFERÊNCIAS}

1. ALENCASTRO MC, et al. Acute intestinal obstruction due to gallstone ileus. Revista do Colégio Brasileiro de Cirurgiões, 2013; 40(4): 275-280.

2. ANSELINE P. Colonic gall-stone ileus. Postgraduate Medical Journal, 1981; 57(663): 62-65.

3. CARLSSON T, GANDHI S. Gallstone ileus of the sigmoid colon: an extremely rare cause of large bowel obstruction detected by multiplanar CT. BMJ case reports, 2015; 2015: bcr2015209654.

4. CHANG L, et al. Clinical and radiological diagnosis of gallstone ileus: a mini review. Emergency radiology, 2018; 25(2): 189-196.

5. CLARO M, et al. Cecal perforation by a large gallstone: an unusual diagnosis. Curēus, 2020; 12(4): e7859.

6. CLAVIEN P, et al. Gallstone lleus. The British journal of surgery, 1990; 77(7): 737-742.

7. CUADRA MB, et al. Colonic gallstone ileus: A rare cause of intestinal obstruction. Cirugía y cirujanos, 2017; 85(5): 440-443.

8. FARKAS $\mathrm{N}$, et al. A systematic review of gallstone sigmoid ileus management. Annals of medicine and surgery, 2018; 27: 32-39.

9. HALABI WJ, et al. Surgery for gallstone ileus: a nationwide comparison of trends and outcomes. Annals of surgery, $2014 ; 259(2): 329-35$.

10. INUKAI K, et al. Sigmoid gallstone ileus: a case report and literature review in Japan. International journal of surgery case reports, 2018; 49: 51-54.

11. INUKAI K. Gallstone ileus: a review. BMJ open gastroenterology, 2019; 6(1): e000344.

12. LAXAGUE F, et al. Pathophysiology, Diagnosis, and Treatment of Colonic Gallstone lleus in an Elderly Patient. ACG case reports journal, 2020; 7(4): e00363.

13. LEE CH, et al. Gallstone ileus with jejunum perforation managed with laparoscopic-assisted surgery: rare case report and minimal invasive management. International surgery, 2015; 100(5): 878-881.

14. OSMAN N, et al. Gallstone ileus of the sigmoid colon: an unusual cause of large-bowel obstruction. HPB surgery : a world journal of hepatic, pancreatic and biliary surgery, 2010; 2010: 153740.

15. PÉREZ JM, et al. Jejunal diverticular perforation due to gallstone ileus. Revista española de enfermedades digestivas : organo oficial de la Sociedad Española de Patología Digestiva, 2012; 104(9): 503-505.

16. RANGA N. Large bowel and small bowel obstruction due to gallstones in the same patient. BMJ Case Reports, 2011; 2011: bcr0920103372.

17. REISNER RM, COHEN JR. Gallstone ileus: a review of 1001 reported cases. The American surgeon, 1994; 60(6): 441-446.

18. RIGLER LG, et al. Gallstone obstruction: pathogenesis and roentgen manifestations. JAMA, 1941; 117(21): 17531759.

19. SCHOOFS C, et al. Cholecystocolonic fistula complicated by gallstone impaction and perforation of the sigmoid. Belgian journal of radiology, 2010; 93(1): 32.

20. TAN YM, et al. A comparison of two surgical strategies for the emergency treatment of gallstone ileus. Singapore medical journal, 2004; 45(2): 69-72. 